\title{
Estudo genético-quantitativo de medidas lineares de eqüinos da raça Mangalarga Marchador - I. Estimativas dos fatores de ambiente e parâmetros genéticos
}

\section{Genetic-quantitative study of body measurements of Mangalarga Marchador horse bred in Brazil - I. Estimates of genetic and environmental effects}

\author{
Luiz Cesar Zamborlini' ${ }^{1}$, José Aurélio Garcia Bergmann², Carmen Silva Pereira², \\ Cleusa Graça Fonseca², Adalgisa S.R. Carneiro²
}

\section{Resumo}

Embora o interesse em pesquisas sobre morfologia em algumas espécies tenha sido menor do que aquele proporcionado aos estudos sobre a produtividade, as características em eqüinos são importantes por estarem intrinsecamente relacionadas à sua funcionalidade de aptidão.

Desta forma, os objetivos do presente estudo foram os de estimar herdabilidades e correlações fenotípicas e genéticas entre doze medidas corporais provenientes dos Livros de Registro Genealógicos da Associação Brasileira dos Criadores do Cavalo Mangalarga Marchador, entre 1967 e 1992, por ocasião do Registro Genealógico de 29.076 animais. As estimativas foram efetuadas pelo método dos quadrados mínimos, através do programa Harvey (1990). Os efeitos fixos de ano, idade à avaliação e sexo foram significativos para a maioria das medidas estudadas. Estimativas de herdabilidade apresentam valores de moderados $(0,40$ para o perímetro torácico) a altos $(0,80$ para o comprimento do dorso), com um valor médio para as doze medidas corporais de 0,56 , evidenciando a importância do componente genético aditivo nas características morfológicas dos animais Mangalarga Marchador. As correlações genéticas entre as características variaram de $-0,09$ (perímetro da canela - comprimento do dorso) a 0,96 (altura na cernelha - altura na garupa). Sessenta e três das 66 correlações possíveis $(95,5 \%)$ apresentaram sinal positivo e destas, $40(60,6 \%)$ apresentaram valor igual ou superior a 0,30 . Este achado demonstra o sinergismo existente entre a maioria das características estudadas.

Palavras chave: melhoramento genético; eqüinos; Mangalarga Marchador

\section{Introdução}

Os eqüinos introduzidos no Brasil foram, na sua maio- ria, originários da Península lbérica onde as raças Árabe e Barbo eram predominantes. Além destas, o Alter e o Andaluz foram as raças de maior influência na formação do cavalo de sela do Brasil. Das raças que se desenvolveram na Região Sudeste do país, a Mangalarga, a Mangalarga Marchador e a Campolina foram as que mereceram maior destaque como animais de sela.

O Padrão Racial da raça Mangalarga Marchador foi estabelecido em 1950; a partir daquela data os animais têm sido avaliados por técnicos credenciados pela Associação Brasileira dos Criadores de Cavalos Mangalarga Marchador (ABCCMM) no que diz respeito à sua conformação e qualidade como animais de sela, principalmente em relação ao seu andamento. Adicionalmente à tomada da medida de diversas partes do corpo dos animais, são atribuídas notas, de acordo com a observação visual do técnico, a componentes corporais e às suas proporções, levando-se em consideração o comprimento, a profundidade, o balanço, a harmonia e a simetria de desenvolvimento de todas as regiões do animal.

Estudos sobre a caracterização racial, baseados em dados biométricos das raças nacionais, são escassos. $O$ presente trabalho visa contribuir para a identificação dos fatores de ambientes e dos parâmetros genéticos que influenciam a conformação da raça Mangalarga Marchador.

\section{Material e Métodos}

Os daidos foram obtidos dos Livros dos Registros Genealógicos mantidos pela ABCCMM $(1991,1992)$, referentes a registros realizados de 1967 a 1992, perfazendo um total inicial de 35.599 animais. As mensurações foram efetuadas com hipômetro metálico e fita métrica, utilizando-se como referência anatômica os pontos citados por Brito (1990) e Barbosa (1993). Com o objetivo de reduzir o erro amostral das estimativas das componentes de variância, foram incluídos nas análises apenas aque-

\footnotetext{
1 Departamento de Zootecnia, Faculdade de Veterinária, UFF, Caixa Postal 100086, 24230-340 Niterói, RJ, Brasil, E-mail: mzozamb@ vm..uff.br

2 Departamento de Zootecnia, Faculdade de Veterinária, UFMG, Caixa Postal 567, 30161-970 Belo Horizonte, MG, Brasil
} 
les garanhões que tiveram pelo menos três progênies, reduzindo-se a amostra a 29.076 animais. Para formação de arquivo e análise descritiva dos dados foi utilizado o programa SAS - Statistical Analysis System (1986).

As análises tiveram como objetivo estimar, simultaneamente, os efeitos de ambiente e os parâmetros genéticos das medidas corporais e da pontuação obtida por ocasião do Registro Genealógico Definitivo. A utilização do modelo proposto foi decidida de acordo com as informações disponíveis nos dados originais e com os resultados obtidos nas análises de consistência prévias. As estimativas da componente de variância foram obtidas pelo Método III de Henderson (1953), utilizando-se, para obtenção das análises, o programa de computador desenvolvido por Harvey (1990). Como as análises preliminares não constataram interações entre os efeitos principais, foi utilizado o seguinte modelo misto:

$Y_{i j k}=u+a_{i}+F_{j}+e_{i j k}$

onde:

$\mathrm{Y}_{\mathrm{ijk}}=$ variável dependente (medida corporal);

$\mathrm{u}=$ média geral;

$a_{i}=$ grupo de efeitos aleatórios (garanhão);

$F_{i}=$ grupo de efeitos fixos (ano, sexo e idade à avaliação);

$e_{i j \mathrm{k}}=$ erro aleatório associado a cada observação.

Visando a verificação do enquadramento da raça no Sistema Eclético de Proporções de Lesbre (1920), procedeu-se a uma análise estatística dos principais quocientes esperados $(\mathrm{Qe})$ calculada a partir das medidas corporais observadas em relação ao quociente observado (Qo). Foi utilizado o teste "t", com $t=Q e-Q o / \sigma_{\overline{(x)}}, e s_{\overline{(x)}}=\sigma / n^{1 / 2}$, sendo $n$ o número de observações e $\sigma_{\overline{(x)}}$ o erro-padrão da média.
As herdabilidades e as correlações genéticas e fenotípicas foram estimadas através da análise de meioirmãos paternos, de acordo com Falconer (1960).

Todas as análises foram realizadas em computador IBM-4381 do Núcleo de Processamento de Dados da Universidade Federal Fluminense (NPD/UFF) e em microcomputador 486 DX2 da Faculdade de Veterinária da UFF.

\section{Resultados e Discussão}

\section{Descrição dos dados}

A descrição das medidas corporais estudadas, referentes aos 29.076 animais é apresentada nas Tabelas $1 \mathrm{e}$ 2. Esses resultados são mais uma contribuição à caracterização de animais da raça Mangalarga Marchador e estão de acordo com os resultados obtidos por Barbosa (1993).

Os resultados dos cálculos dos quocientes esperados (Qe), quocientes obervados (Qo), os erros padrão da média e respectivos valores do teste "t" estão resumidos na Tabela 3.

Todos os Qo das medidas corporais diferem dos $\mathrm{Qe}$ $(P<0,01)$. Os testes de significância para quocientes são muito sensíveis e os quocientes ideais dos Sistema Eclético são frutos de observações de vários autores e aperfeiçoado por Lesbre (1920), especialmente para a raça Árabe.

\section{Fontes de variações de ambiente}

Os efeitos do sexo, ano e idade à época da avaliação foram altamente significativos em todas as medidas corporais, exceto para o comprimento da cabeça, quando o

Tabela 1 - Descrição numérica e estatística das medidas corporais e avaliação por tabela de pontos de 29.076 animais da raça Mangalarga Marchador nos dois sexos

\begin{tabular}{lcccc}
\hline Variáveis & Média & Desvio padrão & Valor mínimo & Valor máximo \\
\hline Tabela de pontos & 73,60 & 4,99 & 60,00 & 95,00 \\
Altura na cernelha & 1,46 & 0,03 & 1,38 & 1,58 \\
Altura na garupa & 1,46 & 0,03 & 1,37 & 1,57 \\
Largura da cabeça & 0,20 & 0,005 & 1,17 & 0,24 \\
Largura da anca & 0,50 & 0,01 & 0,40 & 0,57 \\
Perímetro torácico & 1,72 & 0,06 & 1,53 & 1,95 \\
Perímetro da canela & 0,18 & 0,01 & 0,15 & 0,21 \\
Comprimento da cabeça & 0,57 & 0,02 & 0,49 & 0,65 \\
Comprimento do pescoço & 0,60 & 0,03 & 0,50 & 0,75 \\
Comprimento do dorso & 0,56 & 0,06 & 0,40 & 0,80 \\
Comprimento da garupa & 0,50 & 0,02 & 0,39 & 0,62 \\
Comprimento da espádua & 0,53 & 0,03 & 0,44 & 0,66 \\
Comprimento do corpo & 1,48 & 0,04 & 1,33 & 1,64 \\
\hline
\end{tabular}


Tabela 2 - Descrição numérica e estatística das medidas corporais e avaliação por tabela de pontos de acordo com o sexo dos animais da raça Mangalarga Marchador

\begin{tabular}{|c|c|c|c|c|c|c|c|c|}
\hline \multirow[b]{2}{*}{ Variáveis } & \multicolumn{4}{|c|}{5.470 Machos $(18,8 \%)$} & \multicolumn{4}{|c|}{23.606 Fêmeas $(81,2 \%)$} \\
\hline & Média & $\begin{array}{l}\text { Desvio } \\
\text { padrão }\end{array}$ & $\begin{array}{l}\text { Valor } \\
\text { máximo }\end{array}$ & $\begin{array}{l}\text { Valor } \\
\text { mínimo }\end{array}$ & Média & $\begin{array}{l}\text { Desvio } \\
\text { padrão }\end{array}$ & $\begin{array}{l}\text { Valor } \\
\text { máximo }\end{array}$ & $\begin{array}{r}\text { Valor } \\
\text { mínimo }\end{array}$ \\
\hline Tabela de pontos & 75,54 & 4,89 & 60,00 & 95,00 & 73,14 & 4,90 & 60,0 & 95,00 \\
\hline Alt. cernelha & 1,50 & 0,024 & 1,41 & 1,58 & 1,45 & 0,032 & 1,38 & 1,58 \\
\hline Alt. garupa & 1,49 & 0,024 & 1,42 & 1,57 & 1,45 & 0,031 & 1,37 & 1,57 \\
\hline Larg. cabeça & 0,20 & 0,008 & 0,17 & 0,24 & 0,20 & 0,008 & 0,17 & 0,24 \\
\hline Larg. anca & 0,50 & 0,018 & 0,41 & 0,57 & 0,50 & 0,019 & 0,40 & 0,57 \\
\hline P. torácico & 1,74 & 0,049 & 1,53 & 1,95 & 1,72 & 0,057 & 1,53 & 1,95 \\
\hline P. canela & 0,18 & 0,008 & 0,15 & 0,21 & 0,17 & 0,006 & 0,15 & 0,21 \\
\hline Comp. cabeça & 0,58 & 0,017 & 0,49 & 0,65 & 0,57 & 0,016 & 0,49 & 0,65 \\
\hline Comp. pescoço & 0,62 & 0,030 & 0,50 & 0,75 & 0,60 & 0,026 & 0,50 & 0,75 \\
\hline Comp. dorso & 0,57 & 0,070 & 0,40 & 0,80 & 0,55 & 0,061 & 0,40 & 0,80 \\
\hline Comp. garupa & 0,52 & 0,022 & 0,40 & 0,62 & 0,50 & 0,021 & 0,39 & 0,62 \\
\hline Comp. espádua & 0,55 & 0,031 & 0,44 & 0,66 & 0,52 & 0,028 & 0,44 & 0,66 \\
\hline Comp. corpo & 1,51 & 0,035 & 1,33 & 1,64 & 1,48 & 0,038 & 1,33 & 1,63 \\
\hline
\end{tabular}

Tabela 3 - Comparação das proporções do cavalo Mangalarga Marchador como o Sistema Eclético nos dois sexos

\begin{tabular}{lllllll}
\hline Variáveis & $\begin{array}{l}\text { Quociente } \\
\text { esperado } \\
(\mathrm{Qe})\end{array}$ & $\begin{array}{l}\text { Quociente } \\
\text { observado } \\
(\mathrm{Qo})\end{array}$ & $\begin{array}{l}\text { Erro } \\
\text { padrão da } \\
\text { média }\end{array}$ & Teste "t" & $\begin{array}{l}\text { Proporção } \\
\text { ideal }\end{array}$ & $\begin{array}{l}\text { Proporção } \\
\text { observada }\end{array}$ \\
\hline Alt. cernelha & 1,42 & 1,46 & 0,00021 & $190,48^{*}$ & $1: 2,5$ & $1: 2,57$ \\
Alt. garupa & 1,42 & 1,46 & 0,00020 & $200,00^{*}$ & $1: 2,5$ & $1: 2,57$ \\
Larg. cabeça & 0,19 & 0,20 & 0,00005 & $204,08^{*}$ & $1: 3$ & $1: 3,5$ \\
Comp. pescoço & 0,57 & 0,60 & 0,00016 & $187,50^{*}$ & $1: 1$ & $1: 1,06$ \\
Comp. dorso & 0,47 & 0,56 & 0,00037 & $243,24^{*}$ & $5: 6$ & $5: 6,7$ \\
Comp. garupa & 0,47 & 0,50 & 0,00013 & $230,76^{*}$ & $5: 6$ & $5: 6,1$ \\
Comp. espádua & 0,57 & 0,53 & 0,00018 & $222,22^{*}$ & $1: 1$ & $1: 0,92$ \\
Comp. corpo & 1,42 & 1,48 & 0,00023 & $260,87^{*}$ & $1: 2,5$ & $1: 2,6$ \\
Índice corporal & & & & & 0,86 & 0,85 \\
Índice dáctilo-torácico & & & & & 0,108 & 0,103 \\
\hline
\end{tabular}

* $(P<0,01)$

efeito da idade à avaliação não mostrou significância. $O$ sexo do animal mostrou-se um fator importante na variação das medidas corporais e nas avaliações por tabela de pontos, com valores maiores para machos. Os resultados deste estudo concordam com os obtidos por Arnason $(1979,1984)$ que observou que o sexo e o ano da avaliação afetavam consideravelmente todas as medidas corporais da raça Icelandic Toelter na Islândia. O efeito do ano da avaliação, embora de difícil explicação, pode ser atribuído a variações anuais na qualidade da alimentação, no manejo geral e nos critérios de avaliação adotados pelos técnicos do Registro Genealógico. Similarmente, a idade à avaliação foi uma importante fonte de variação nas medidas corporais estudadas. Portanto, a avaliação e a seleção de animais baseadas nas medidas de conformação devem ser precedida de correções para estes fatores.

\section{Estimativas dos parâmetros genéticos e fenotípicos}

As estimativas de herdabilidade, correlações genéticas e fenotípicas e seus respectivos erros padrão, obtidos pela correlação intraclasse entre meio-irmãos paternos, podem ser observados na Tabela 4.

As estimativas de herdabilidade das características de conformação apresentam valores de moderados $(0,40$ para o perímetro torácico) a altos ( 0,80 para o comprimento do dorso), com um valor médio para as doze medidas corporais de animais Mangalarga Marchador e a possibilidade de se obterem mudanças genéticas nas populações através da seleção massal. Também a avaliação pela tabela de pontos, que pode ser considerada até certo ponto arbitrária, apresentou um importante componente genético aditivo: herdabilidade igual a 0,62. 
Tabela 4 - Estimativas de herdabilidade (diagonal), correlações genéticas (acima da diagonal) e fenotípicas (abaixo da diagonal) das medidas corporais na da raça Mangalarga Marchador

\begin{tabular}{|c|c|c|c|c|c|c|c|c|c|c|c|c|}
\hline Medidas & 1 & 2 & 3 & 4 & 5 & 6 & 7 & 8 & 9 & 10 & 11 & 12 \\
\hline Alt. cernelha & 0,49 & 0,96 & 0,14 & 0,61 & 0,45 & 0,23 & 0,47 & 0,29 & 0,13 & 0,51 & 0,34 & 0,80 \\
\hline Alt. garupa & 0,93 & 0,48 & 0,15 & 0,58 & 0,39 & 0,19 & 0,54 & 0,30 & 0,20 & 0,51 & 0,37 & 0,80 \\
\hline Larg. cabeça & 0,16 & 0,16 & 0,52 & 0,187 & 0,20 & 0,03 & 0,22 & 0,33 & 0,33 & $-0,05$ & 0,30 & 0,24 \\
\hline Larg. anca & 0,43 & 0,42 & 0,20 & 0,52 & 0,49 & 0,29 & 0,46 & 0,30 & 0,28 & 0,51 & 0,34 & 0,70 \\
\hline P. torácico & 0,40 & 0,37 & 0,18 & 0,42 & 0,40 & 0,45 & 0,19 & 0,38 & 0,02 & 0,30 & 0,26 & 0,52 \\
\hline P. canela & 0,27 & 0,24 & 0,09 & 0,26 & 0,31 & 0,43 & 0,06 & 0,02 & $-0,09$ & 0,20 & 0,19 & 0,26 \\
\hline Comp. cabeça & 0,40 & 0,41 & 0,19 & 0,34 & 0,22 & 0,09 & 0,60 & 0,41 & 0,32 & 0,51 & 0,70 & 0,54 \\
\hline C. pescoço & 0,35 & 0,33 & 0,20 & 0,24 & 0,28 & 0,14 & 0,39 & 0,56 & 0,31 & 0,17 & 0,37 & 0,48 \\
\hline Comp. dorso & 0,14 & 0,17 & 0,26 & 0,15 & 0,03 & 0,00 & 0,23 & 0,26 & 0,80 & $-0,03$ & 0,23 & 0,32 \\
\hline C. garupa & 0,41 & 0,41 & 0,07 & 0,48 & 0,32 & 0,17 & 0,37 & 0,25 & 0,09 & 0,61 & 0,59 & 0,53 \\
\hline C. espádua & 0,35 & 0,34 & 0,20 & 0,35 & 0,30 & 0,13 & 0,44 & 0,25 & 0,21 & 0,19 & 0,78 & 0,41 \\
\hline Comp. corpo & 0,70 & 0,69 & 0,20 & 0,50 & 0,41 & 0,26 & 0,42 & 0,39 & 0,26 & 0,48 & 0,39 & 0,52 \\
\hline
\end{tabular}

As correlações genéticas entre características variaram de $-0,09$ (correlação entre o perímetro da canela e o comprimento do dorso) a 0,96 (correlação entre as alturas na cernelha e na garupa). De todas as 66 correlações possíveis, $63(95,5 \%)$ apresentaram sinal positivo e, destas, $40(60,6 \%)$ apresentaram valor igual ou superior a 0,30 . Este achado demonstra o sinergismo existente entre a maioria das características estudadas. Com este estudo fica evidenciado que as mesmas respondem à seleção. Entretanto, faz-se necessária uma definição, por parte de técnicos e criadores, de que características seriam importantes para o desempenho da função de animais da raça Mangalarga Marchador. Uma vez definido o papel (ou importância) de cada característica morfológica, considerando as herdabilidades e as correlações genéticas, seria conveniente a utilização de índices de seleção para um programa de melhoramento genético. Deve-se salientar que, embora o interesse sobre a morfologia em algumas espécies tenha sido menor do que aquele proporcionado aos estudos sobre a produtividade, as características morfológicas em eqüinos são importantes por estarem intrinsecamente relacionadas à sua funcionalidade e aptidão.

\section{Conclusões}

1. As fontes de variações de ambientes, ano, idade à avaliação e sexo afetaram significativamente as medidas corporais estudadas. Desta forma, deve-se levar em consideração essas fontes de variação quando se for estimar o mérito genético aditivo dos animais.

2. O Sistema Eclético de proporções foi aplicado, revelando-se a raça Mangalarga Marchador mediolínea, de acordo com os índices corporal de 0,86 e dáctilo-torácico de 0,103 para ambos os sexos, não se enquadrando nas proporções desse Sistema.
3. Os valores das proporções lineares deverão ter continuidade através de avaliações complementares das proporções angulares dos raios ósseos das principais articulações envolvidas com o tipo de andamento da raça Mangalarga Marchador.

\section{Abstract \\ Genetic-quantitative study of body measurements of Mangalarga Marchador horse bred in Brazil - I. Esti- mates of genetic and environmental effects}

The objective of this study was to estimate heritabilities, genetic and phenotypic correlation of twelve body measurements of the Brazilian horse bred Mangalarga Marchador. Records from 29,076 animals, measured between 1967 and 1992, were analyzed by Least-Square and Maximum Likelihood Computer Program (Harvey, 1990). The fixed effect of year, age, and sex of foal were signifcant for most of the characteristics. Heritability estimates varied between 0.40 (thoracic diameter) to 0.80 (back length), with a medium value of 0.56 , characterizing the importance of the genetic component of body measurements in horses. Genetic correlations varied between -0.09 (cannon diameter - back length) and 0.96 (withers height). Sixty-three of the 66 possible correlations (95.5\%) were positive; $40(60.6 \%)$ were superior to 0.30 . It was evident that selection for one (or combination) of traits would have effect on almost all other morphological traits.

Key words: animal breeding; horses

\section{Agradecimentos}

À Superintendência do Registro Genealógico da Associação Brasilera dos Criadores de Cavalo Mangalarga Marchador pela cessão dos dados. 


\section{Referências bibliográficas}

ARNASON, Th. Studies on traits in the Icelandic Toelter Horses. I. Estimation of some environmental effects and genetic parameters. Agric. Res. v. 111, p. 81-92, 1979.

ARNASSON, Th. Genetic studies on conformation and performance of Icelandic Toelter Horse. Acta Agric. Scand., v. 34, p. 409-427, 1984.

ASSOCIAÇÃO BRASILEIRA DOS CRIADORES DO CAVALO MANGALARGA MARCHADOR. A história do cavalo Mangalarga Marchador, Belo Horizonte: Nova Fronteira, 1991, 89p.

ASSOCIAÇÃO BRASILEIRA DOS CRIADORES DO CAVALO MANGALARGA MARCHADOR. Estatuto. Belo Horizonte: $O$ Lutador, 1992, 65p.

BARBOSA, C.G. Estudo morfométrico na raça Mangalarga Marchador: uma abordagem multivariada. Belo Horizonte, 1993, 76p. Tese (mestrado) - Escola de Veterinária. Universidade Federal de Minas Gerais.
BRITO, A.A. Avaliação e mensuração. In: ASSOCIAÇÃO DOS CRIADORES DO CAVALO DA RAÇA MANGALARGA MARCHADOR. Capacitação de técnicos e árbitros da raça Mangalarga Marchador. Belo Horizonte: 1990, 123p.

FALCONER, D.S. Introduction to Quantitative Genetics, (2 ed.) Edinburgh: Oliver \& Boyd, 1960, 430p.

HARVEY, W.R. User's Guide for LSMLMW; Mixed Model Least Squares and Maximum Likelihood Computer Program. PC2 version. Ohio State University, 1990, 100p.

HENDERSON, C.R. Estimation of variance and covariance components. Biometrics, jun., p. 226-252, 1953.

HENDERSON, C.R. Sire evaluation and genetic trends. In: Proc. Anim. Breeding and Genetic Symp. in Honor of Dr Jay L. Lush. ASAS-ADSA, 1973.

LESBRE, F.X. Precis d'Exterieur du Cheval. Paris: Freres Ed., 1920

SAS. User's Guide: Statistics. 5 ed., Cary, North Carolina: SAS Institute Inc. 1986, 956p. 